\title{
Scission-induced bounds on maximum polymer drag reduction in turbulent flow
}

\author{
Siva A. Vanapalli and Mohammad T. Islam \\ Department of Chemical Engineering, University of Michigan, Ann Arbor, Michigan 48109 \\ Michael J. Solomon \\ Department of Chemical Engineering and Macromolecular Science and Engineering Program, \\ University of Michigan, Ann Arbor, Michigan 48109
}

(Received 22 November 2004; accepted 12 July 2005; published online 26 September 2005)

\begin{abstract}
We report the direct quantification of molar mass degradation in the drag-reducing polymers polyethylene oxide (PEO) and polyacrylamide (PAM) in turbulent pipe flows with an upstream tapered contraction. We find that entrance effects associated with the upstream contraction dominate the polymer degradation. Quantifying degradation according to the scaling relationship $\dot{\gamma}_{w} \propto M_{\mathrm{ws}}^{-n}$, the exponent $n$ is determined to be $-2.20 \pm 0.21$ and $-2.73 \pm 0.18$ for PEO and PAM, respectively. Here $M_{\mathrm{ws}}$ is the steady-state (or limiting) weight-average scission molar mass. A methodology is devised to circumvent polymer degradation due to the upstream contraction and thereby conduct degradation experiments in which only the turbulent flow in the pipe is responsible for chain scission. In this case, the scission-scaling relationship for PEO is $\dot{\gamma}_{w} \propto M_{w}^{-3.20 \pm 0.28}$. Here $M_{w}$ is the degraded weight-average molar mass after one pass through the 1.63-m length of pipe. Based on these scaling relationships we obtain a new upper limit for polymer drag reduction that is determined by chain scission rather than the maximum drag reduction asymptote. (C) 2005 American Institute of Physics. [DOI: 10.1063/1.2042489]
\end{abstract}

\section{INTRODUCTION}

The addition of a few parts per million of highmolecular weight polymer leads to dramatic decrease in frictional losses in turbulent pipe flows. ${ }^{1,2}$ This phenomenon, referred to as polymer turbulent drag reduction, has generated significant interest because of potential technological applications such as fast ocean transport and efficient pipeline transport of fluids. ${ }^{3-5}$

According to Virk's phenomenology, ${ }^{6}$ the simplest way to achieve maximum drag reduction (MDR) for a given polymer-solvent system is to use high concentrations of high-molecular weight polymer. Yet, there are two practical limitations to this approach. First, cost considerations typically require low polymer concentrations for large-scale applications. Second, high-molecular weight polymers undergo chain scission at the high Reynolds numbers characteristic of practical turbulent flows. ${ }^{7-11}$ Molecular degradation of highmolecular weight polymers lowers the drag-reducing capability of the polymer chains and therefore limits the amount of drag reduction that can be achieved.

Early investigations of polymer degradation conducted experiments in pipe flows. ${ }^{7-11}$ More recently, behavior in rotational flows such as the Taylor Couette and rotating disk geometries has been studied. ${ }^{12-15}$ Experiments in rotational flows have the advantage of requiring less fluid volume. They are also well suited to characterize polymer degradation kinetics. ${ }^{13,14,16-18}$ However, despite significant advances in the aforementioned geometries, ${ }^{13,19}$ to our knowledge no framework exists to quantitatively describe the bounds that polymer chain scission places on the maximum drag reduction that can be attained in turbulent pipe flow. By the mea- surement of absolute molar mass distributions under a range of conditions in turbulent pipe flow, this study provides this framework for the common drag-reducing agents poly(ethylene oxide) and polyacrylamide. Moreover, there is a correspondence between local conditions in pipe flow, turbulent flow in a square channel, and turbulent flow in a developing boundary layer that yields an expectation that results from the former geometry will be locally applicable to the latter geometries. $^{20-22}$

Molecular weight, polymer concentration, solvent quality, turbulent intensity, and flow geometry have been identified as important factors influencing polymer degradation in turbulent pipe flows. ${ }^{7-11,23}$ In particular, it has been reported that high molar mass polymers break preferentially relative to low molar mass chains $8,9,11$ and that scission occurs predominantly at the chain midpoint. ${ }^{24}$ In addition, it has been found that the degree of degradation is correlated with the turbulent intensity of the flow. ${ }^{10,11}$ Most studies to date have used indirect methods such as changes in friction factor and intrinsic viscosity ${ }^{8,11,25}$ to assess polymer degradation. In some cases the molar mass distributions of degraded polymers have been measured using gel permeation chromatography with refractive index detection and calibration with standards of known molar mass. ${ }^{7,9,10,23}$ Because of the development of light-scattering detectors, absolute characterization of polymer molar mass distribution by gel permeation chromatography is today possible. ${ }^{26-29}$ Here we apply this technique to measure the absolute molar mass distribution of polymer molecules that have been subjected to turbulent flow. To our knowledge, this is the first such characterization of polymer degradation in turbulent pipe flow. 
Most previous work studied polymer degradation at turbulent flow conditions that fall near the MDR asymptote. ${ }^{8,9,11,23}$ Yet, because of the asymptotic nature of the MDR regime, changes in molecular weight and concentration have little detectable effect on frictional drag near MDR. ${ }^{6}$ As a result, changes due to polymer degradation are difficult to resolve, especially with the indirect method based on friction factor measurement. ${ }^{11}$ To address these difficulties, here we study degradation in the polymeric regime, ${ }^{6}$ where friction drag and chain scission depend upon the Weissenberg number $\left(\mathrm{We} \equiv \lambda u_{\tau}^{2} / \nu\right.$, where $\lambda$ is the relaxation time of the polymer, $u_{\tau}$ is the friction velocity, and $\nu$ is the kinematic viscosity of the solvent. Here, $u_{\tau} \equiv \sqrt{\tau_{w} / \rho}$, where $\tau_{w}$ is the wall shear stress and $\rho$ is the solvent density) and the Virk slope increment $\delta\left(\sim M_{w} \downarrow c\right.$, where $M_{w}$ is the weightaverage molar mass of the polymer and $c$ is the concentration of polymer molecules). The quantities We and $\delta$ are highly sensitive to polymer molecular weight and concentration.

Another complication associated with studying polymer degradation in turbulent pipe flows is entrance effects. Experimentally, turbulent pipe flow setups inevitably possess an upstream contraction. The upstream contraction is needed to minimize polymer degradation in the flow loop outside the test section. However, the upstream contraction is itself a possible source for chain scission because flow through it contains an extensional component. Because polymer degradation studies in turbulent pipe flows typically involve very high wall shear rates $\left(\sim 10^{5} \mathrm{~s}^{-1}\right)$, the extensional strain rates in the upstream contraction can be high enough to induce polymer degradation. Indeed, studies have shown that the contraction geometry very efficiently degrades polymer molecules. ${ }^{30-32}$ This implies that polymer chains could break in the entrance region before they experience the fully developed turbulent flow. The current literature ${ }^{7,8,11}$ is unclear on which of the two regions dominate polymer degradation. Here we seek to resolve the entrance effect issues that might pervade the polymer degradation literature published to date.

In this paper we generate a scaling relationship between the applied wall shear rate and the polymer molar mass distribution that results due to the turbulent flow. Such relationships have been developed for extensional flows: ${ }^{28,33,34} \dot{\varepsilon}$ $\propto M_{w}^{-2}$ and $\dot{\varepsilon} \propto M_{w}^{-1}$ have been quantified in stagnation-point flows and contraction flows, respectively, where $\dot{\varepsilon}$ is the characteristic strain rate for scission. Further, these scaling exponents, particularly in the stagnation-point flow, are sensitive to the inertial character of the flow. ${ }^{28}$ However, to our knowledge, no such relationships exist for turbulent flows which are essentially driven by inertia. In addition to the fundamental relevance of this scaling to polymer dynamics in turbulent flows, the relationship will allow definition of an operating window for drag reduction in which polymer chains will reduce friction drag but not undergo scission. Identification of this operating regime will be immediately applicable to the selection of polymer chain architecture, concentration, and molar mass for practical implementation of friction drag technologies.

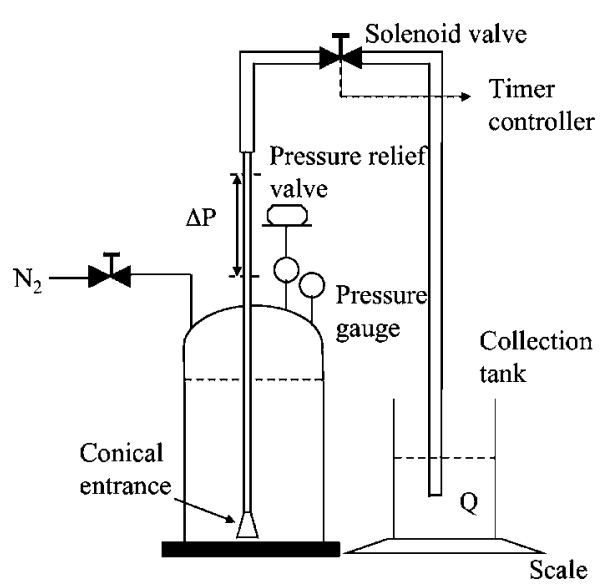

FIG. 1. Schematic diagram of the experimental turbulent pipe flow apparatus.

\section{EXPERIMENT}

\section{A. Polymer preparation}

Two common drag-reducing water-soluble polymerspolyethylene oxide (PEO, Polysciences, Warrington, PA) and polyacrylamide (PAM, Hyperfloc, Hychem, Tampa, FL) were chosen for this study. The nominal molecular weights reported by the manufacturer for these polymer samples are $5 \times 10^{6}$ and $15 \times 10^{6} \mathrm{~g} / \mathrm{mol}$, respectively. De-ionized water was used as the solvent for all the experiments.

The following procedure was used to prepare the polymer solutions. Stock solutions at concentrations of 1000 2000 ppm (parts per million) were prepared in de-ionized water. To prevent shear degradation, polymer dissolution was performed in 1- or 2-1 containers rotated at 3-6 rpm on a rolling apparatus for 24-48 h (Wheaton Science Products, Millville, NJ). Samples were then diluted to the desired concentrations and experiments were conducted within a day of their dilution.

Certain studies in the literature indicate that the PEO molecules may potentially aggregate under quiescent conditions. ${ }^{35,36}$ In our case, a dynamic light-scattering analysis of the PEO used in the study did not reveal long-time relaxations in the intensity autocorrelation function that are characteristic of aggregates (data not shown). This observation agrees with results reported by several other authors that PEO does not necessarily aggregate under quiescent conditions. ${ }^{37-39}$ Moreover, it is likely that aggregates, if at all present, are disrupted into individual molecules due to the high shear rates in the turbulent flow. Thus, data reported here are indicative of the properties of individual polymer chains.

\section{B. Turbulent flow apparatus}

A schematic diagram of the experimental apparatus used for conducting polymer chain scission experiments in turbulent pipe flow is given in Fig. 1. Stainless-steel pipes [inside diameter (i.d.) $=10.9 \mathrm{~mm}$ ] of several different lengths $(0.3$, $1.27,1.68,2.12$, and $3.0 \mathrm{~m}$ ) were used to conduct the turbulent flow experiments. To prevent degradation of polymer chains outside the test section and to achieve high wall shear 
rates, a pressure-driven flow apparatus was used to convey the fluid through the test section. A 16-gal carbon steel pressure vessel (Alloy Products Inc., Waukesha, WI) was used as a constant pressure reservoir. The vessel was hydraulically connected to a compressed nitrogen gas cylinder through a precision gas regulator to maintain constant pressure $(<5 \%$ deviation) during experimentation. To minimize deleterious extensional flow components generated due to the sudden contraction prior to the test section, the fluid was allowed to enter the test section from the reservoir through a tapered contraction with an area ratio of 21.6:1 and an included angle of $24^{\circ}$. This angle was chosen based on the degradation studies done by Nguyen and Kausch ${ }^{32}$ in contraction flows.

Two differential pressure transducers (GP50, Grand Island, NY, error $\pm 0.02 \mathrm{psi}$ ) with range from $0-10$ to $0-50 \mathrm{psi}$ were used to obtain precise pressure drop measurements. Here we define the entrance length as the distance between the exit of the conical entrance and beginning of the first pressure tap. The second pressure tap was always located 85 $\mathrm{cm}$ from the first pressure tap in all our experiments. The entrance lengths for the experiments with longer tubes$1.68,2.12$, and $3.0 \mathrm{~m}$-are $\sim 73,113$, and 192 pipe diameters, respectively. The longer tubes were used to generate scission-scaling data (cf. Sec. V) and the shorter tubes (0.3 and $1.27 \mathrm{~m}$ ) were used to assess the role of entrance effects on chain scission in turbulent pipe flows (cf. Sec. IV). The pressure transducers were connected to a National Instruments' LABVIEW data acquisition system (Austin, TX). To avoid the possibility of degradation inside the flowmeasuring device, flow rate was measured by collecting samples for a specified amount of time and weighing them. This was accomplished by using a solenoid valve (Magnatrol Valve Corporation, Hawthorne, NJ) connected to a timer controller (National Controls Corp., West Chicago, IL). The flow rate through the test section was controlled by regulating the pressure of the inlet reservoir. After passing through the test section the fluid was allowed to expand into a 21$\mathrm{mm}$-i.d. stainless-steel pipe. This expansion is about twice the diameter of the test section and includes a bend as well. It is possible that polymer chains break in this bend/ expansion. However, here the linear velocity is four times less than in the test section. The lower velocity relative to the upstream contraction and test section indicates that this section is unlikely to affect the steady-state scission scalings (cf. Sec. V) reported in our study.

Experiments were conducted for an inlet pressure range of $3.5 \times 10^{4}-10^{6} \mathrm{~Pa}(5-150 \mathrm{psi})$. For these inlet pressures, the typical Reynolds number $(\operatorname{Re} \equiv U d / \nu$, where $U$ is the mean velocity, and $d$ is the inner pipe diameter) range is $\sim 10^{4}-2.5 \times 10^{5}$. The friction Reynolds numbers $\left(\operatorname{Re}_{\tau}\right.$ $\left.\equiv u_{\tau} d / \nu\right)$ generated in the study range from $\sim 10^{3}$ to $10^{4}$.

\section{Gel permeation chromatography}

The molar mass distributions of degraded PEO and PAM solutions were measured using a gel permeation chromatography (GPC, Waters Corporation, Milford, MA) system in conjunction with refractive index detection and multiangle laser light scattering (MALLS). The solvent $\left(0.1 M \mathrm{NaNO}_{3}\right)$ was filtered $(0.1 \mu \mathrm{m}$, Millipore, Bedford, MA), degassed (Waters in-line degasser), and then pumped (Waters 515 HPLC pump) at a flow rate of $0.2 \mathrm{ml} / \mathrm{min}$. Separation of molar masses was achieved by injecting filtered polymer samples (250- $\mu$ l loop autosampler, Waters 717 Plus) through two temperature-controlled $\left(T=50^{\circ} \mathrm{C}\right)$ columns (Shodex OHpak, SB-806 HQ, SB-804 HQ, Thomson Instruments, Clearbrook, VA).

The radius of gyration and molar mass of the separated fractions was assessed by means of static light scattering (DAWN EOS, Wyatt Technology Corporation, Santa Barbara, CA) of eluted fractions using the Berry formalism. The concentrations of separated fractions were measured using a refractive index (RI) detector (Optilab DSP, Wyatt Technology Corporation, Santa Barbara, CA). The number average $\left(M_{n}\right)$ and weight-average $\left(M_{w}\right)$ molar masses were calculated from the moments of the molar mass distribution using commercial software (ASTRA, Wyatt Technology Corporation). All the molar mass data reported in the figures are weightaverage molar masses.

A study was conducted to assess the performance of the GPC/MALLS system for absolute molar mass determination and the capability of the columns to separate the molar mass fractions of the distribution. This study evaluates possible nonidealities in the GPC such as shear degradation in the columns. For this study, the device was operated in two modes-batch mode and in-line analysis. During in-line analysis, the columns were in place and the weight-average molar mass was calculated from the measured molar mass distribution. During batch mode analysis, the columns were removed and the weight-average molar mass of the polymer sample was measured by using the static light-scattering detector as described by Buchholz and Barron. ${ }^{26}$ Polymer samples of $6-10$ different concentrations $(\sim 10-100 \mathrm{ppm})$ were prefiltered and injected using a syringe pump (Razel Scientific Instruments, Stamford, CT) and the resultant scattering intensities were measured in the range of $26^{\circ}-121^{\circ}$. The Berry formalism [Eq. (1)] with a quadratic angular dependence was used to fit the scattered intensities at concentrations tested to obtain $M_{w}$ and $R_{g}$.

$$
\sqrt{\frac{K c}{R_{\theta}}}=\left(\frac{1}{\sqrt{M_{w}}}+\sqrt{2 A_{2} c}\right)\left(1+\frac{q^{2} R_{g}^{2}}{3}+\cdots\right) .
$$

In the equation given above $K$ is the optical contrast factor, $R_{\theta}$ is the Rayleigh ratio, $A_{2}$ is the second virial coefficient, and $q$ is the scattering vector. ${ }^{40}$

If indeed, the GPC/MALLS system is capable of successfully measuring the true molar mass distributions of polymer samples (and is thus not limited by the aforementioned issues), then the $M_{w}$ obtained from the two independent methods described above should be consistent with each other. As shown in Table I, the data from both approaches agree well (within $\sim 10 \%$ ) and indicate that our GPC/ MALLS system yields absolute molar mass distributions and that moments of those distributions agree with an indepen- 
TABLE I. Moments of the molar mass distribution of the polymers used for the chain scission studies as characterized by GPC-MALLS. PEO1, PEO2, and PAM1 are standards from American Polymer Standards, Mentor, OH. PEO3 was a degraded sample collected after three passes during a turbulent flow experiment at $29000 \mathrm{~s}^{-1}$ in the 3-m-long pipe. PAM2 was obtained from Polysciences, Warrington, PA. The numbers shown in parentheses represent error in estimating the values from fit of the GPC raw data to Berry formalism.

\begin{tabular}{lcrrrrr}
\hline \hline & \multicolumn{2}{c}{ Batch mode analysis } & & \multicolumn{3}{c}{ GPC analysis } \\
\cline { 2 - 3 } Sample & \multicolumn{1}{c}{$M_{w}$} & $R_{z}(\mathrm{~nm})$ & & $M_{w}$ & $R_{z}(\mathrm{~nm})$ & $M_{w} / M_{n}$ \\
\hline PEO1 & $2.15 \times 10^{5}(1.4 \%)$ & $25.3(5 \%)$ & & $1.99 \times 10^{5}(0.5 \%)$ & $23.8(0.3 \%)$ & 1.05 \\
PEO2 & $7.38 \times 10^{5}(1.7 \%)$ & $60.2(2 \%)$ & & $7.81 \times 10^{5}(0.5 \%)$ & $57.2(0.3 \%)$ & 1.00 \\
PEO3 & $2.43 \times 10^{6}(1.8 \%)$ & $145.9(1.2 \%)$ & & $2.57 \times 10^{6}(0.6 \%)$ & $159.0(0.3 \%)$ & 1.40 \\
PAM1 & $1.69 \times 10^{6}(0.6 \%)$ & $65.2(2.5 \%)$ & & $1.46 \times 10^{6}(0.5 \%)$ & $68.7(0.2 \%)$ & 1.44 \\
PAM2 & $4.24 \times 10^{6}(2.9 \%)$ & $128.9(4 \%)$ & $4.77 \times 10^{6}(0.5 \%)$ & $143.6(0.1 \%)$ & 1.28 \\
\hline \hline
\end{tabular}

dent static light-scattering measurement, for the polymers considered here, which have $M_{w}<2.5 \times 10^{6}$ for PEO and $M_{w}<4.25 \times 10^{6}$ for PAM.

In some turbulent flow experiments conducted at low concentrations $(<100 \mathrm{ppm})$, the degraded polymer samples were freeze-dried (Labconco, Kansas City, MO) and then redissolved in the same buffer solution that was used in the GPC analysis. This procedure was tested and found to yield results that agreed with analogous samples that were analyzed directly without freeze-drying (data not shown).

\section{DIRECT QUANTIFICATION OF POLYMER DEGRADATION IN TURBULENT PIPE FLOWS WITH AN UPSTREAM TAPERED CONTRACTION}

Figure 2(a) shows the changes in the turbulent flow friction drag when a 100-ppm PEO solution was passed repeatedly through the turbulent flow setup at a fixed inlet pressure. The percent drag reduction $\left[\% \mathrm{DR} \equiv\left(f_{w}-f_{p}\right) \times 100 / f_{w}\right.$, where $f_{w}$ and $f_{p}$ denote the friction factor in the Newtonian and non-Newtonian turbulent flows] decreases with increasing number of passes. This result is consistent with the degradation of polymer molecules. Further Fig. 2(b) shows that as the Re decreases with the pass number, the wall shear rate increases. Here, we defined $\dot{\gamma}_{w}=\Delta P d / 4 \mu L$, where $\Delta P$ is the pressure drop across the test section of length $L$ and $\mu$ is the solvent viscosity. These observations are in accordance with the qualitative fact that in turbulent flows, polymer degradation leads to a decrease in flow rate or an increase in pressure drop (or wall shear rate) and therefore lowers the drag reduction. Further, note that after about ten passes, all three variables tend to a constant value consistent with steady-state behavior. Similar results were obtained for PAM solutions (data not shown). These results are broadly consistent with prior literature. ${ }^{9,11,18,25}$ [Note the detail that although the overall pressure drop in the device is fixed and independent of pass number, we observe an increase with pass number in the wall shear rate measured locally at the test section. These two seemingly conflicting observations are reconciled by noting that molar mass degradation apparently affects the particular way in which the overall, fixed pressure drop is distributed among the test section, the contraction, and other parts of the flow apparatus. Also note that to estimate wall shear rate we used the solvent viscosity instead of the solu- tion viscosity. At $200 \mathrm{ppm}$ the solution viscosity is $\sim 25 \%$ higher than that of water. However, the solution viscosity itself changes due to polymer degradation and this change is a complex function of the degree of polymer degradation. To
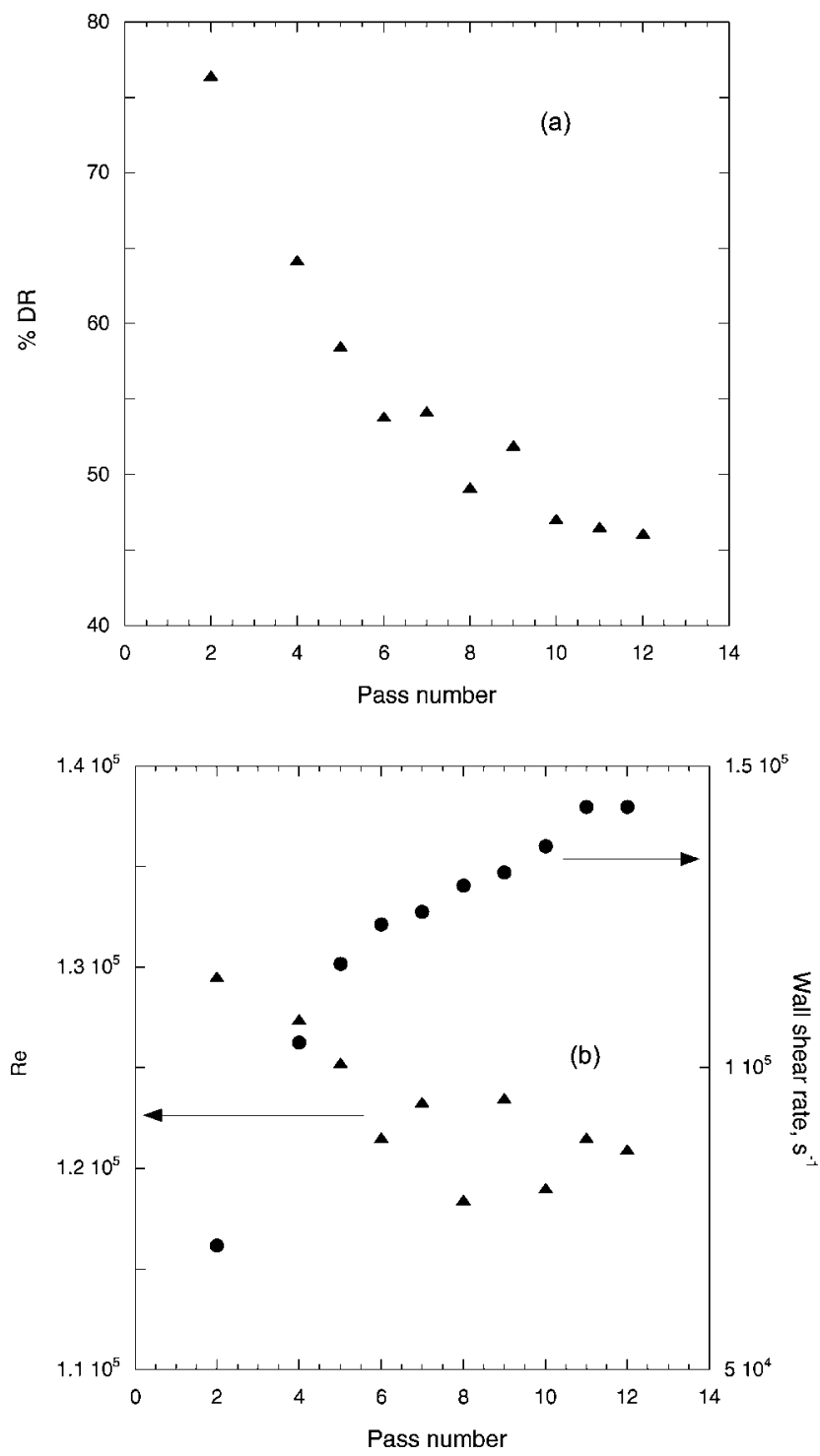

FIG. 2. Changes in the turbulent flow variables with the number of passes for 100-ppm PEO solution in 3-m-long pipe. Note that all the variables reach steady state after a certain number of passes. 

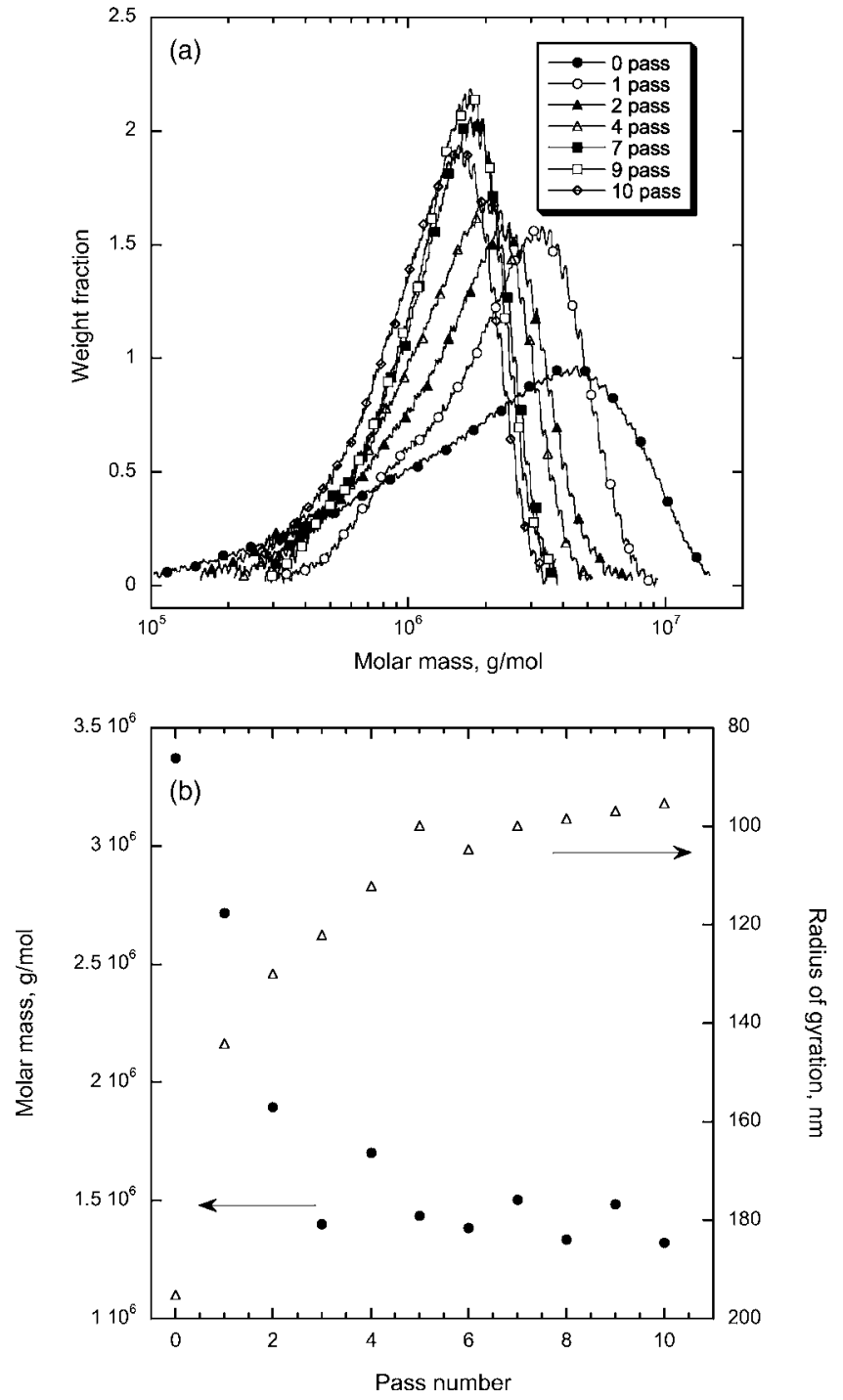

FIG. 3. (a) Evolution of the molar mass distribution with the number of passes for 100-ppm PEO solution in a 3-m-long pipe at Re $\sim 1.2 \times 10^{5}$ and wall shear rate of $\sim 1.45 \times 10^{5} \mathrm{~s}^{-1}$. (b) The corresponding weight-average molar mass $\left(M_{w}\right)$ and radius of gyration $\left(R_{z}\right)$ for each of the distributions as a function of the number of passes. Note that $M_{w}$ and $R_{z}$ reach a constant value after ten passes through the pipe.

avoid this complication and to be consistent among experiments in which polymer concentration and scission history (inlet pressures and number of passes) were varied, we chose to use the solvent viscosity to estimate the wall shear rate.]

Molecular information confirming the degradation process is available from Figs. 3(a) and 3(b). The molar mass distribution of the degraded polymer samples is shown in Fig. 3(a). The molar mass distribution progressively shifts to lower molar mass with increasing pass number. This observation demonstrates that higher-molecular weight chains preferentially degrade. In addition, the molar mass distribution reaches a steady state in about ten passes, in agreement with the steady state in the flow variables discussed above. These data are, to our knowledge, the first absolute quantification of molar mass during polymer degradation in a turbulent pipe flow.

In simple flows, experiments and theory suggest that a critical strain rate is required to break a given polymer chain. ${ }^{31}$ In the present case, apparently not all the polymer chains traversing the pipe flow experience this characteristic strain rate. Thus, only some of the chains break, as shown by the transient data of Fig. 3. However, repeated cycling of the polymer solution progressively increases the probability of breakage. After a number of passes, all chains of molar mass susceptible to breakage have undergone scission. No further changes in molar mass distribution occur and the friction drag behavior attains steady state.

The calculated weight-average molar mass from the distribution and the measured radius of gyration are shown in Fig. 3(b) as a function of the number of passes in the turbulent flow. Both these variables monotonically decrease and attain steady state after about ten passes. The steady-state molar mass is identified as the critical molar mass for scission $\left(M_{\mathrm{ws}}\right)$ that corresponds to the steady-state turbulent wall shear rate. The steady-state molar mass is critical in the sense that there will be no change in this value even with further recycling of the polymer solution.

Experimental studies in extensional flows suggest that intermolecular interactions are important at polymer concentrations far below the overlap concentration. ${ }^{41}$ We evaluated the extent to which scission experiments were being conducted in the dilute regime by using polymer solutions of varying concentration. Our results show that there is negligible influence of polymer concentration on $M_{\mathrm{ws}}$ in spite of changing the concentration by an order of magnitude (cf. Sec. V A).

\section{ASSESSMENT OF ENTRANCE EFFECTS ON POLYMER DEGRADATION IN TURBULENT FLOWS}

From the results of Sec. III alone it is unclear if the contraction flow or the turbulent pipe flow is the primary source of polymer degradation. To investigate the contribution of the entrance geometry to polymer degradation, molar mass distributions were measured as a function of pass number for cases in which the device was equipped with the same upstream tapered contraction but with pipes of different lengths.

Polymer solutions were repeatedly passed through pipes of several different lengths and the molar mass of the degraded samples was measured. If polymer degradation were to arise in both the upstream contraction and the turbulent flow (or, alternatively, just the turbulent flow), then one would expect to see a lower molar mass in solutions that were passed through pipes of longer length. On the other hand, contraction-dominated scission will yield results that are relatively insensitive to the length of the downstream turbulent pipe flow. Figure 4 reports the molar mass as a function of number of passes for pipes of several different lengths at a fixed inlet pressure $(20,50$, and $100 \mathrm{psi})$. Surprisingly, the data show that changing the length of the pipe by an order of magnitude has no measurable effect on the observed molar mass trends. This finding suggests that almost all the degradation in the device occurs in the upstream contraction. Moussa and Tiu arrived at a similar conclusion indirectly by noting changes in frictional drag. ${ }^{11}$ They per- 

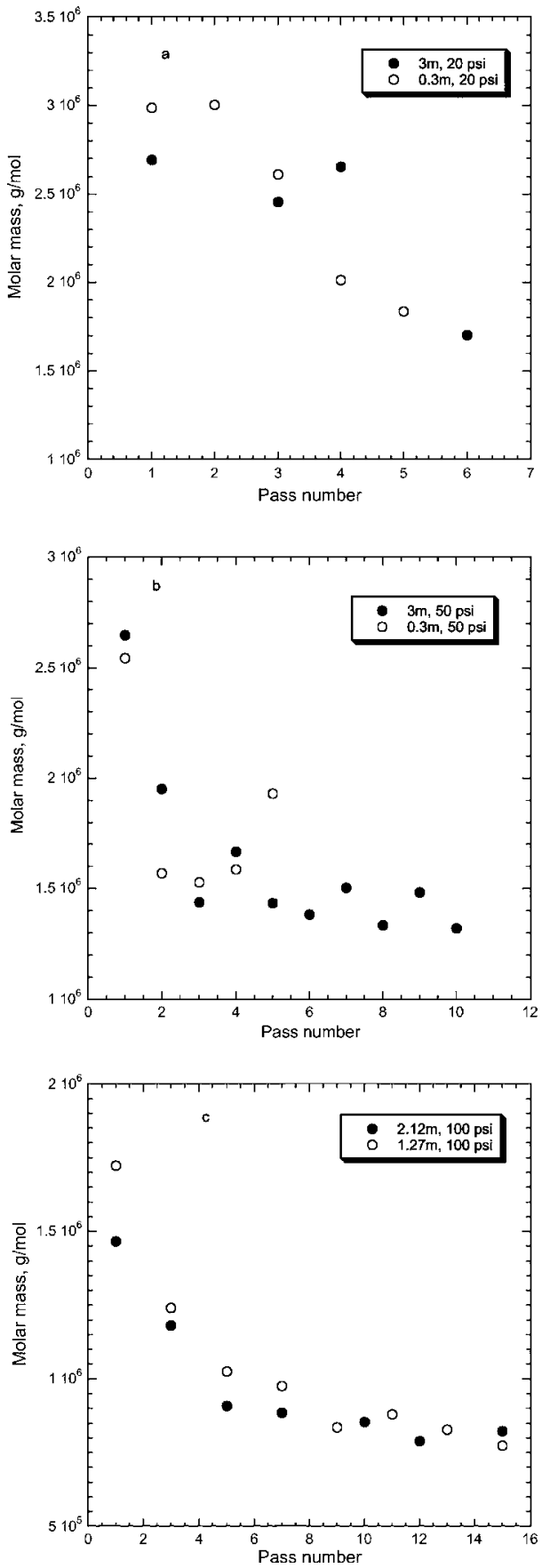

FIG. 4. Assessment of entrance effects in the turbulent pipe flow by changing the lengths of the test section (a) 20, (b) 50, and (c) 100 psi. The pressures in the legend are the inlet pressures used to drive the flow.

formed degradation experiments at various contraction ratios and pipe lengths and found that the entrance effects dominated degradation in the turbulent flows they studied. Taken together, Fig. 4 and Moussa and Tiu ${ }^{11}$ suggest that all earlier reports of polymer degradation in turbulent pipe flow are compromised because such measurements have primarily interrogated scission in the contraction inevitably present upstream of their test sections. These results imply that even in a pipe of arbitrarily long length the upstream contraction will dominate the steady-state scission molar mass since it is a more efficient geometry for scission.

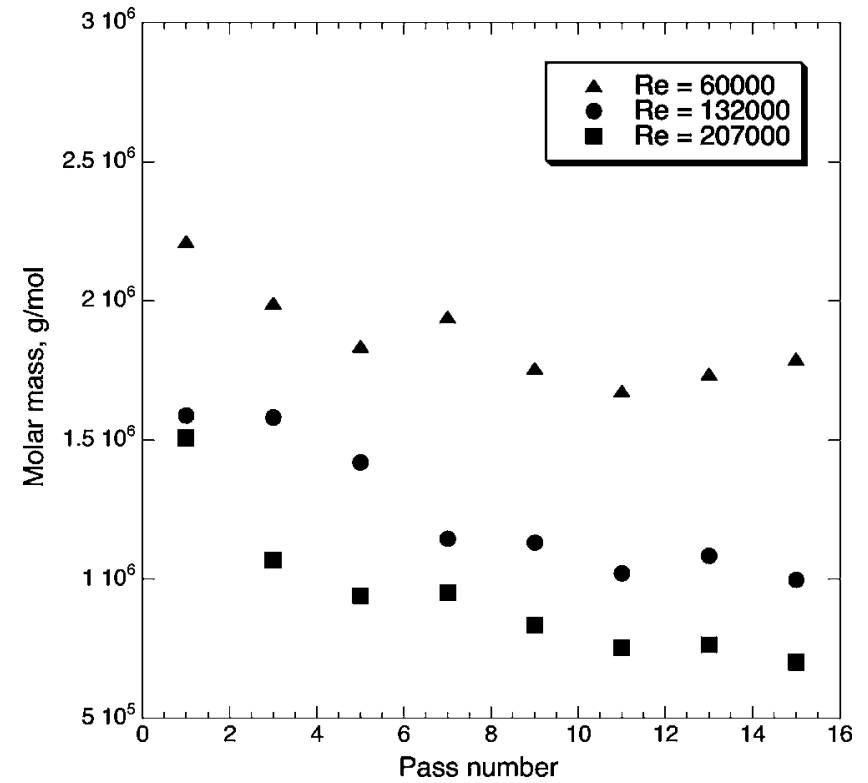

FIG. 5. Experimental results for 100-ppm PEO solution as a function of number of passes for a range of steady-state Reynolds numbers in a 2.12$\mathrm{m}$-long pipe. The corresponding steady-state wall shear rates for the Reynolds number shown in the legend are $4.7 \times 10^{4}, 1.92 \times 10^{5}$, and 4.71 $\times 10^{5} \mathrm{~s}^{-1}$, respectively.

\section{SCISSION-SCALING RELATIONSHIPS IN TURBULENT FLOWS}

\section{A. Scission-scaling relationship in turbulent pipe flow with an upstream tapered contraction}

In Sec. III we introduced the critical molar mass for scission $\left(M_{\mathrm{ws}}\right)$ corresponding to the steady-state wall shear rate of the turbulent flow experiment. Measurements at a range of inlet pressures thus yield a scaling relationship between this turbulent flow variable and this moment of the steady-state molar mass. Figure 5 shows the trends in the molar mass of PEO due to degradation at various inlet pressures each characterized by its steady-state $\operatorname{Re}\left(\operatorname{Re}_{\mathrm{ss}}\right)$. The steady-state molar mass decreases with increasing $\operatorname{Re}_{\mathrm{ss}}$ for this polymer solution.

Now for each $\operatorname{Re}_{\mathrm{ss}}$, we take the turbulent wall shear rate $\dot{\gamma}_{w}$ (at steady state) as the characteristic strain rate measure of the flow. Figure 6 shows the scission-scaling relationships for PEO and PAM solutions as $\dot{\gamma}_{w} \propto M_{\mathrm{ws}}^{-2.20 \pm 0.21}$ and $\dot{\gamma}_{w}$ $\propto M_{\mathrm{ws}}^{-2.73 \pm 0.18}$, respectively, generated from the data like Fig. 5 (analogous data for PAM not shown). It is apparent from the plot that for the same molar mass PEO is more susceptible to degradation than PAM. This quantitative result agrees with previous qualitative reports. ${ }^{42,43}$ Figure 6 also shows that the scalings for the two polymers are independent of polymer concentration. In addition, the data shown in the scaling relationship include measurements from all the three longer tubes (possessing different entrance lengths) and they do not deviate significantly from the overall established trend. This suggests that the entrance lengths do not affect our chain scission-scaling results. We believe Fig. 6 to be the first quantification of the relationship between polymer molar mass and turbulent flow intensity (with an upstream tapered contraction present). The two polymers, PEO and PAM, are 


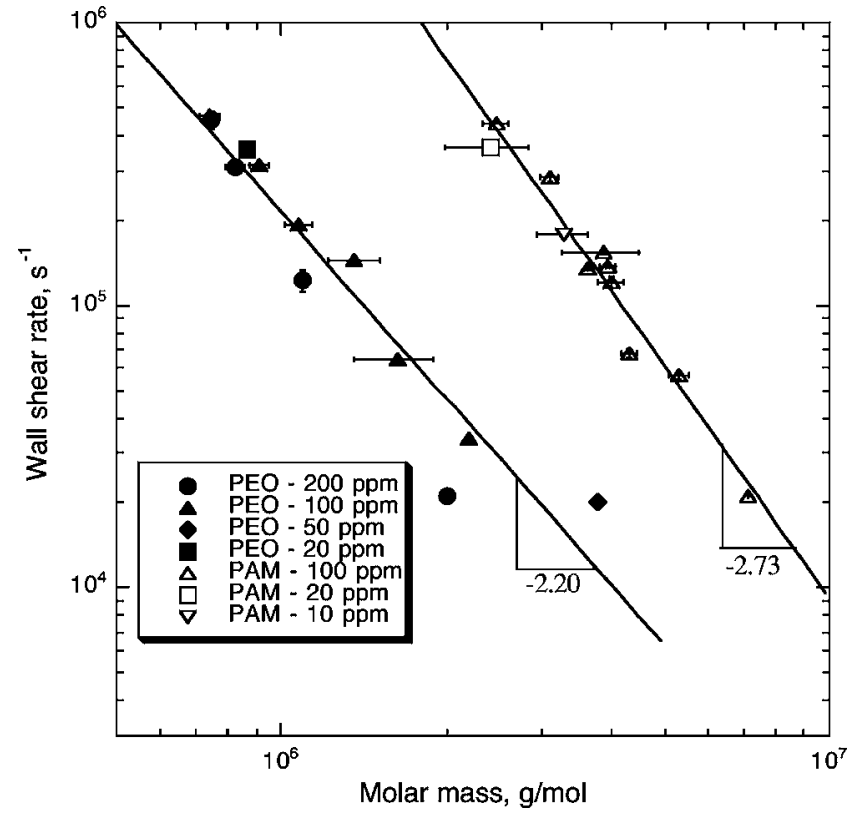

FIG. 6. Scaling relationship between wall shear rate and molar mass of the polymer in turbulent pipe flow. The closed symbols are for PEO and the open symbols are for PAM. The straight lines are power-law fits to the experimental data points with scaling exponents of $-2.20 \pm 0.21$ for PEO and $-2.73 \pm 0.18$ for PAM, respectively. The error bars are shown for both the wall shear rate and molar mass as standard deviations of the experimental data (the error bars for wall shear rates are considerably smaller than that of the molar mass and are hard to see visually).

widely used in turbulent drag reduction studies. Although these scalings were developed under conditions where degradation occurs predominantly in the entrance region, they are still applicable to the prediction of the practical performance of drag-reducing polymers (cf. Sec. VI) in turbulent pipe flows because upstream contractions are inevitably present in turbulent pipe flow studies.

Now, we reanalyze the Fig. 6 data to evaluate the hypothesis that scission is determined by the characteristic strain rate, $\dot{\varepsilon} \cong U_{\text {ss }} / d$, in the upstream contraction. Here, $U_{\text {ss }}$ is the mean velocity corresponding to $\mathrm{Re}_{\mathrm{ss}}$ and we omit from the definition an $O(1)$ prefactor that depends on the contraction conical angle. ${ }^{31}$ The reanalysis yields the following scalings for PEO and PAM, respectively (Fig. 7): $\dot{\varepsilon} \propto M_{\mathrm{ws}}^{-1.23 \pm 0.12}$ and $\dot{\varepsilon} \propto M_{\mathrm{ws}}^{-1.27 \pm 0.16}$. The two scaling exponents are almost identical to each other and in approximate agreement with the previously observed scaling exponent for polymer scission in contraction flows $\left(\dot{\varepsilon} \propto M_{\mathrm{ws}}^{-1.0}\right){ }^{33}$ This remarkable result implies that the elongational strain rate in the upstream contraction dictates the steady-state molar mass in the whole turbulent flow experiment. This finding further supports our earlier claim that existing literature on polymer degradation in turbulent pipe flows must be reevaluated in light of the profound effect of an upstream contraction on turbulent scission results. On the practical side, these scission relationships are needed to design components of large-scale polymer drag reduction studies such as injectors, polymer delivery systems, and sampling ports since these devices all possess contractions.

\section{B. Scission-scaling relationship in a purely turbulent pipe flow}

Thus, in turbulent pipe flows entrance effects dominate scission and any contraction upstream sets a characteristic strain rate that determines the steady-state scission product distribution. Although, this finding requires reevaluation of previous studies of polymer degradation in turbulent pipe flows, ${ }^{7,8,10,11}$ it does not imply that the fluctuating strain rates in a turbulent flow field are insufficient to break polymer chains. Indeed, measurements in a rotating apparatus nominally devoid of entrance effects have shown that polymer chains do undergo scission in turbulent flows. ${ }^{13,14}$ Note, however, that the characteristics of the turbulent flow in these devices could be very different from that in pipe flows that are of practical relevance.

In seeking to design an experiment that truly addresses scission in turbulent pipe flows, one possibility is to optimize the geometry of the upstream contraction to minimize degradation at the entrance. However, studies by Moussa and Tiu, ${ }^{11}$ in both tapered and abrupt contractions of several different contraction ratios, have shown that this approach will not be successful. Furthermore, in a different study, focused exclusively on contraction flows of various geometries, Nguyen and Kausch ${ }^{32}$ (see Fig. 6 in the reference) found that the mean velocity at the entrance is the sole factor controlling the extent of degradation and that degradation is largely independent of the details of the contraction geometry. These studies, in conjunction with our own results, suggest that changing the contraction geometry will not alleviate the entrance effect. Instead, we have adopted the alternative approach of circumventing the upstream contraction to conduct scission experiments in a purely turbulent pipe flow.

The approach that we adopted to decouple entrance effects and thereby study true turbulent shear flow-induced scission involves loading (at low shear rates) a plug of polymer solution into the device so that it is located downstream of the region in which entrance effects occur. The entrance region is filled with the solvent plug that adjoins the polymer plug. The whole assembly is then driven through the device by the application of a specified inlet pressure. To conduct this experiment the existing turbulent flow apparatus was modified with the valve assembly of Fig. 8. To conduct the polymer-plug experiment we execute five steps.

First, the pressure vessel is initially filled with the desired amount of solvent (water) and then pressurized (with valves $B$ and $C$ closed) such that the solvent level is above valve A. Second, valve A is closed and the excess solvent above it drained by opening valve B (and keeping valve C closed). Step two creates a solvent plug of length $0.97 \mathrm{~m}$ $(L / D \sim 89)$. Third, valve B is closed and (with valve A also closed) a 200-ppm polymer solution is loaded into the pipe through valve C. Taking care that all air is removed and that the entire column above valve A is filled with polymer solution, a polymer plug of length $1.63 \mathrm{~m}(L / D \sim 150)$ is created. Fourth, valve $\mathrm{C}$ is closed, the solvent tank is pressurized to the target value, and valve A is opened. Finally, in a fifth step, the solenoid valve is released and the pressure drop measured across a test section of length $0.127 \mathrm{~m}$. The time 


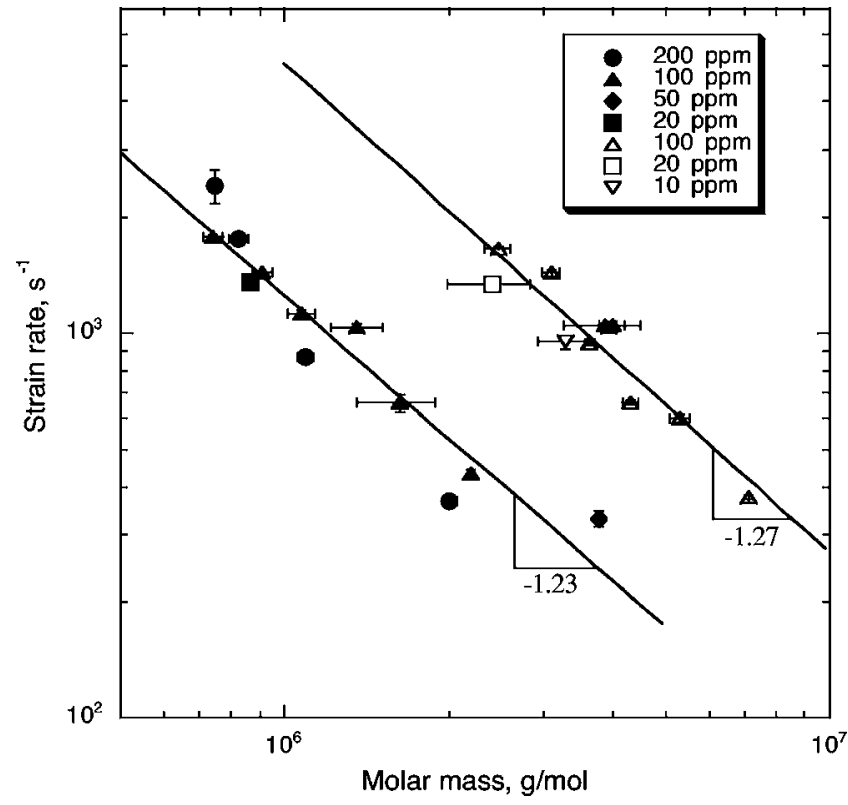

FIG. 7. Scaling relationship between strain rate and molar mass of the polymer in the entrance region of the turbulent flow. The closed symbols are for PEO and the open symbols are for PAM. The straight lines are powerlaw fits to the experimental data points with scaling exponents of $-1.23 \pm 0.12$ for PEO and $-1.27 \pm 0.16$ for PAM, respectively. The error bars are shown for both the strain rate and molar mass as standard deviations of the experimental data (the error bars for strain rates are considerably smaller than that of the molar mass and are hard to see visually).

interval between steps 4 and 5 was always less than $5 \mathrm{~s}$. (Pressure drop measurements made with a solvent-solvent plug combination differed negligibly from those involving a polymer-solvent plug combination.) Samples were collected at the end of each run and freeze-dried for GPC analysis.

Since the polymer plug does not traverse the contraction, any degradation that occurs must be due to the turbulent shear flow only. Results of the PEO plug experiments after one pass through $1.63 \mathrm{~m}$ of pipe are reported in Fig. 9. The scaling exponent is $-3.20 \pm 0.28$. This value is significantly greater than that measured in the turbulent flow with en-

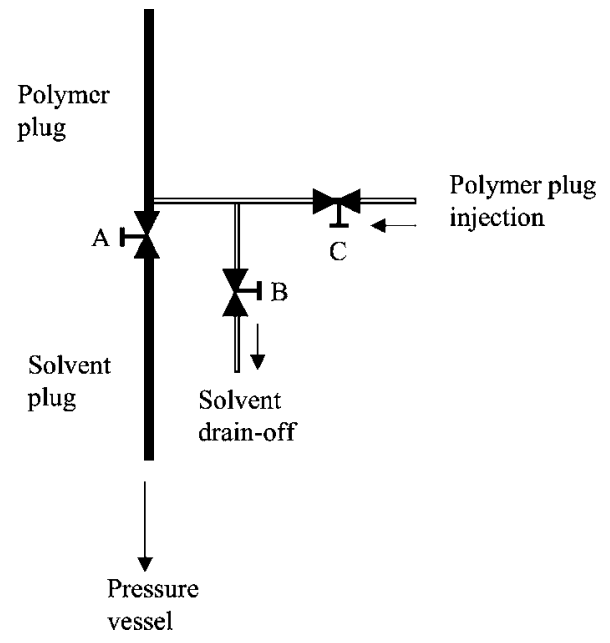

FIG. 8. Schematic diagram of the valve assembly used to perform scission experiments using the polymer slug approach.

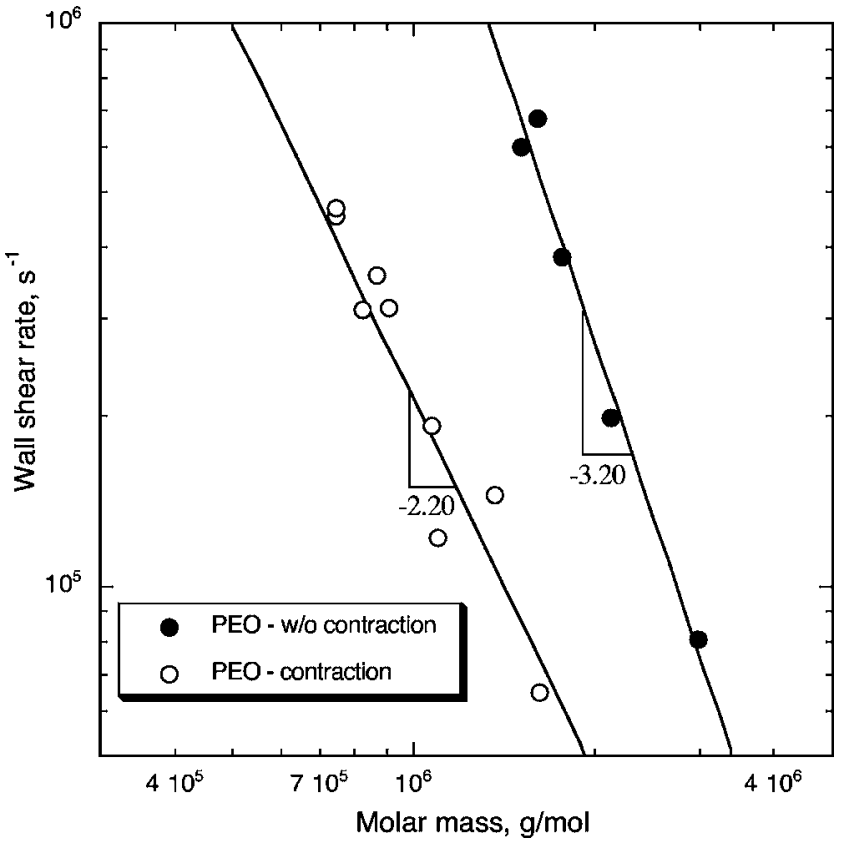

FIG. 9. Scaling relationship for scission between wall shear rate and molar mass of the polymer after one pass through $1.63-\mathrm{m}$ pipe in the PEO polymer-plug experiment (filled circles). The straight line is a power-law fit to the experimental data points with scaling exponents of $-3.20 \pm 0.28$. The scission data for PEO from Fig. 6 in which contraction dominates degradation are replotted for comparison (open circles).

trance effects (cf. Fig. 6). In addition to a different scaling, the curves have different prefactors, since the pure turbulent flow curve is shifted relative to the turbulent flow with contraction data. The analogous scaling exponent for PAM is not available because the pure turbulent shear flow generated in our device was not sufficiently strong to degrade the chains to molar masses low enough to be characterized by our GPC system. We further note that after one pass through the pure turbulent flow, the mean molar mass $\left(M_{w}=2.1\right.$ $\times 10^{6} \mathrm{~g} / \mathrm{mol}$ ) is higher than that obtained from turbulent pipe with tapered contraction flow $\left(M_{w}=1.7 \times 10^{6} \mathrm{~g} / \mathrm{mol}\right)$ under identical experimental conditions $\left(\dot{\gamma}_{w}=2 \times 10^{5} \mathrm{~s}^{-1}, L\right.$ $=1.63 \mathrm{~m})$. This observation supports our earlier claim that the upstream contraction dominates degradation in turbulent pipe flows.

We acknowledge certain nonidealities possible for these scission experiments in a pure turbulent shear flow. First, when the solenoid valve is opened and the turbulent flow is generated, mixing of the two adjoining plugs may occur. However, this mixing is likely minimal because the polymer and solvent plugs at turbulent velocities will have little opportunity for axial mixing due to the short duration of the experiment. Second, the residence time of polymer molecules in the pipe is not uniform due to the finite length of the polymer plug. This situation differs from the experiments of Sec. III where all polymer molecules experienced the same residence time. Furthermore, since the polymer plug is not passed through the pipe multiple times, some molecules may remain unbroken even though their molar mass is great enough to ultimately break if the plug was repeatedly cycled through the device. 


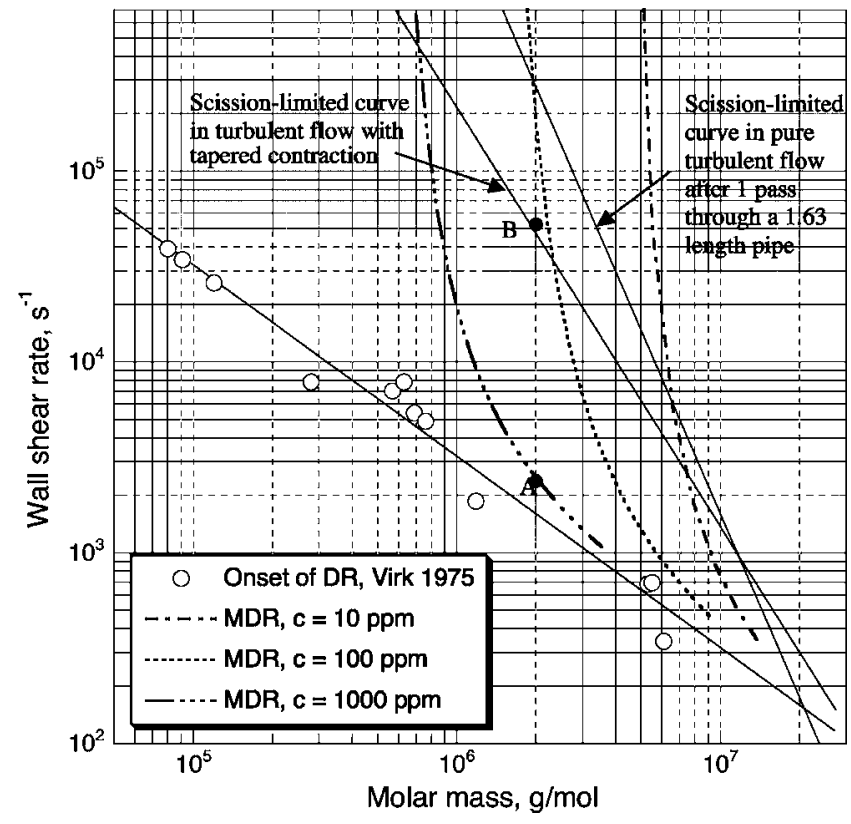

FIG. 10. Bounds on polymer drag reduction for PEO due to chain scission on a wall shear rate basis.

The presence of these nonidealities implies that the scission molar masses reported in Fig. 9, after one pass in the pipe, may not be steady-state molar masses, or the true critical scission molar mass that corresponds to the measured wall shear rate. Therefore the scaling exponent of $-3.20 \pm 0.28$ extracted from these experiments should be considered as only a preliminary relationship describing the scission of polymer molecules in a truly turbulent shear flow. Nevertheless, the relationship is immediately applicable to the estimate of scission bounds on maximum drag reduction as we consider in Sec. VI.

\section{BOUNDS ON POLYMER DRAG REDUCTION IN TURBULENT PIPE FLOWS DUE TO CHAIN SCISSION}

In this section, we consider the scission-scaling relationships obtained in Sec. $\mathrm{V}$ and discuss their implications for polymer turbulent drag reduction. In particular, we use the scission relationships to generate upper bounds on the drag reduction that can be achieved for a given polymer molecular weight and concentration. In order to achieve these limits, we apply the Virk's phenomenological ${ }^{6}$ correlations linking polymer molecular parameters to drag reduction.

For flexible polymers, the onset of drag reduction occurs at a critical wall shear rate $\left(\dot{\gamma}_{w}^{*}\right)$. Below $\dot{\gamma}_{w}^{*}$, the PrandtlKarman (PK) coordinates follow the PK law for Newtonian solvents in turbulent flows. From the experimental data of PEO given by Virk $^{6}$ we deduce the following relationship relating the onset wall shear rate to molecular weight (see Fig. 10):

$$
\dot{\gamma}_{w}^{*}=3.35 \times 10^{9} M_{w}^{-1} .
$$

Once this critical shear rate is exceeded, there is a decrease in friction factor $\left(f \equiv 2 \tau_{w} / \rho U^{2}\right)$ with increase in Reynolds number, which can be represented as

$$
1 / \sqrt{f}=(4.0+\delta) \log _{10}(\operatorname{Re} \sqrt{f})-0.4-\delta \log _{10}\left[(\operatorname{Re} \sqrt{f})^{*}\right]
$$

where $\operatorname{Re} \sqrt{ } f^{*} \equiv \sqrt{ } 2 u_{\tau}^{*} d / \nu\left(u_{\tau}^{*}\right.$ is the friction velocity at the onset of drag reduction). The magnitude of the slope increment depends on the type of polymer used and increases with molecular weight and concentration of the polymer. For example, for $\mathrm{PEO}$,

$$
\delta=1.242 \times 10^{-6} c^{0.5} M_{w} .
$$

Of course, with further increase in Re, \%DR does not increase indefinitely. The limiting behavior is the maximum drag reduction asymptote given by

$$
1 / \sqrt{f_{\mathrm{MDR}}}=19.0 \log _{10}(\operatorname{Re} \sqrt{f})_{\mathrm{MDR}}-32.4 \text {. }
$$

The MDR asymptote is strikingly insensitive to polymer concentration, type, and molecular weight.

We now identify upper limits on drag reduction due to chain scission. Figure 10 reports the onset correlation of drag reduction [Eq. (2)] along with the measured scission-scaling relationships. The two scission curves intersect each other at $\dot{\gamma}_{w} \sim 1000 \mathrm{~s}^{-1}$ and $M_{w} \sim 10^{7} \mathrm{~g} / \mathrm{mol}$. In addition, Fig. 10 shows three other curves labeled MDR for $c=10 \mathrm{ppm}, \mathrm{MDR}$ for $c=100 \mathrm{ppm}$, and MDR for $c=10000 \mathrm{ppm}$. These curves represent the locus of intersection of the polymeric regime lines and the MDR asymptote for a given polymer concentration and pipe diameter $(10.9 \mathrm{~mm})$. The locus was obtained by solving Eqs. (3) and (5) for various $M_{w}$ and a specific polymer concentration $(c$, in ppm). As shown in the plot, the calculated MDR curves for all the three polymer concentrations-10, 100, and 1000 ppm-intersect the scission-scaling curves. The intersection of these curves identifies certain regions in concentration, molar mass, and wall shear rate space for which drag reduction due to PEO molecules will be ultimately limited by scission dynamics rather than the MDR asymptote.

Figure 10 defines the operating window for optimal drag reduction of PEO molecules of any molar mass. For example, consider a PEO solution of $M_{w}=2 \times 10^{6} \mathrm{~g} / \mathrm{mol}$. At a concentration of $1000 \mathrm{ppm}$, Fig. 10 shows that this polymeric liquid reaches MDR at $\dot{\gamma} \sim 2000 \mathrm{~s}^{-1}$ (point $A$ in Fig. 11). As the wall shear rate of a turbulent pipe flow is increased, the drag reduction will thus encounter a MDR asymptote limitation before it encounters either scission limitation curve. However, if a concentration of 100 ppm were instead studied, Fig. 10 shows that the molecules would break at $\dot{\gamma} \sim 50000 \mathrm{~s}^{-1}$ (point $B$ in Fig. 10). This critical wall shear rate for breakage would be encountered before MDR would be attained. That is, at this concentration, the maximum attainable drag reduction is limited by scission and not by the MDR. Therefore, Fig. 10 gives quantitative practical limits on maximum drag reduction achievable for a given polymer, taking into account the polymer parameters (molecular weight and concentration) and operating conditions (pipe diameter, Re, and wall shear rates). This operating regime for drag reduction is one of the principal findings of this study.

To illustrate these limitations of scission in the PrandtlKarman coordinate notation that is common in the literature, 


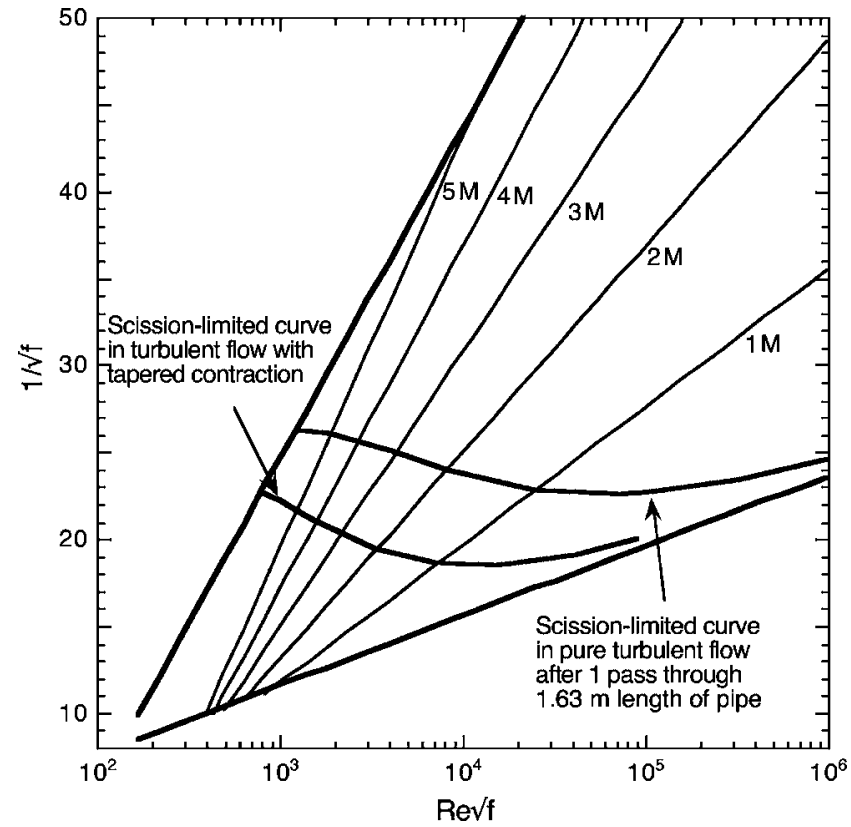

FIG. 11. Bounds on polymer drag reduction for PEO due to chain scission on a Prandtl-Karman plot. The scission curves are drawn for polymer concentrations of $10 \mathrm{ppm}$. The unit $M$ denotes $1 \times 10^{6} \mathrm{~g} / \mathrm{mol}$.

the data shown in Fig. 10 have been translated into these coordinates in Fig. 11. The plot shows the drag reduction curves for hypothetical PEO solutions with $M_{w}=(1-5)$ $\times 10^{6} \mathrm{~g} / \mathrm{mol}$ and concentration $c=10 \mathrm{ppm}$. These curves were generated by using Eqs. (2)-(4). Here the curves have been drawn for a pipe i.d. $=10.9 \mathrm{~mm}$ (which corresponds to the pipe used in our turbulent flow setup). The plot also shows the scission-scaling curves redrawn on these coordinates. Figure 11 clearly demonstrates that the drag reduction for 10-ppm PEO solutions of commercially available molar mass is scission limited below the MDR. Note that in PK coordinates, the scission curves are a function of polymer concentration. With increasing polymer concentration they shift to the right. Therefore, as is intuitively plausible, it is possible to reach MDR before becoming scission limited by increasing the polymer concentration. Also note that the drag reduction curves on $\mathrm{PK}$ coordinates are a function of pipe diameter. For those seeking to apply Fig. 11 to other pipe flow geometries of differing diameter, Fig. 11 may be rescaled accordingly, i.e., since $\operatorname{Re} \sqrt{ } f \equiv \sqrt{ } 2 u_{\tau} d / \nu$, for a fixed friction velocity (or wall shear stress), the curves shift to the right in an amount proportional to the increase in pipe diameter.

To test if the scission limits proposed in Fig. 11 are indeed obeyed by practically relevant drag-reducing agents we used a PEO of $M_{w} \sim 2 \times 10^{6} \mathrm{~g} / \mathrm{mol}$ (WSR-N60K, Dow Chemical Co., Midland, MI) at a concentration of $20 \mathrm{ppm}$. This polymer solution was tested in the turbulent flow apparatus with the upstream tapered contraction (described in Sec. II B). The skin-friction data are shown in Fig. 12 and each datum point represents a fresh polymer solution. Also shown is the expected drag reduction curve for this polymer (at $20 \mathrm{ppm}$ ) using Virk's literature correlation. First, note that the experimental data are in good agreement with the Virk

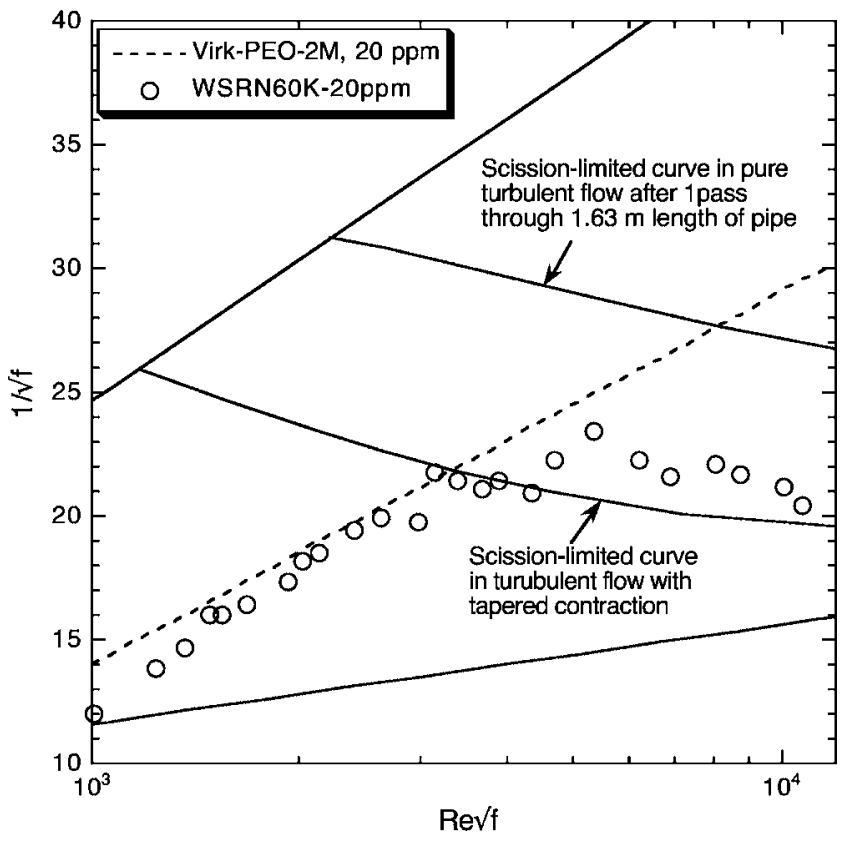

FIG. 12. Experimental verification of the bounds on PEO drag reduction due to chain scission on a Prandtl-Karman plot. The hollow circles represent friction data collected for 20 ppm of WSR-N60K (Dow Chemical Co., Midland, MI; $\left.M_{w} \sim 2 \times 10^{6} \mathrm{~g} / \mathrm{mol}\right)$ in the turbulent flow setup with the upstream contraction. The dotted line represents the estimated slope increment from Virk's phenomenology. The scission curves are drawn for polymer concentrations of $20 \mathrm{ppm}$.

correlation in the polymeric regime. However, the drag reduction curve for this polymer increasingly deviates from its predicted slope increment for $\operatorname{Re} \sqrt{ }>\sim 3000$. This point of departure coincides very well with the scission limit independently generated using the scaling relationship developed for turbulent flows with an upstream tapered contraction. The correspondence suggests that scission-limited bounds are indeed encountered under conditions relevant to polymer turbulent drag reduction.

Taken together, Figs. 10-12 indicate that Virk's phenomenological correlations are not capable of predicting drag reduction at high wall shear rates. However, the scission scalings reported in this study can be used to assess the upper limits to which they will be predictive. Further Figs. 10 and 11 may be used by practitioners to predict and mitigate scission effects in situations where polymers are used for friction drag reduction.

\section{CONCLUSIONS}

The degradation experiments reported here, in turbulent pipe flows with a gradual upstream contraction, demonstrate that entrance effects dominate polymer chain scission in typical turbulent pipe flow devices. Measured scaling relationships have been used to generate upper bounds on polymer turbulent drag reduction that are due to chain scission. The availability of these limits on polymer drag reduction will allow the pragmatic selection of operating conditions for friction drag reduction. On the theoretical side, the scission scalings may be incorporated into direct numerical simula- 
tions of turbulent flows ${ }^{44,45}$ to obtain accurate and quantitative estimates of polymer drag reduction.

\section{ACKNOWLEDGMENTS}

We acknowledge Ramana Vishnubhotla, Dean Malmgren, and Robert Glied for help with experiments. We thank Professor Ronald Larson and Anshuman Roy for useful discussions. This research was supported by DARPA under Contract No. MDA972-01-1-0020.

${ }^{1}$ B. A. Toms, Proceedings of the First International Congress on Rheology (North-Holland, Amsterdam, 1949), Vol. 2, p. 135.

${ }^{2}$ P. S. Virk and E. L. Mollo-Christensen, "The Toms phenomenonturbulent pipe flow of dilute polymer solutions," J. Fluid Mech. 30, 305 (1967).

${ }^{3}$ R. G. Larson, "Analysis of polymer drag reduction on a flat plate," J. Non-Newtonian Fluid Mech. 111, 229 (2003).

${ }^{4}$ E. D. Burger, L. G. Chron, and T. K. Perkins, "Studies of drag reduction conducted over a broad range of pipe line conditions when flowing Prudhoe Bay crude oil," J. Rheol. 24, 603 (1980).

${ }^{5}$ R. H. J. Sellin, J. W. Hoyt, J. Pollert, and O. Scrivener, "The effect of drag-reducing additives on fluid flows and their industrial applications. Part 2: Present applications and future proposals," J. Hydraul. Res. 20, 235 (1982)

${ }^{6}$ P. S. Virk, "Drag reduction fundamentals," AIChE J. 21, 625 (1975).

${ }^{7}$ J. Culter, J. L. Zakin, and G. K. Patterson, "Mechanical degradation of dilute solutions of high polymers in capillary tube flow," J. Appl. Polym. Sci. 19, 3235 (1975).

${ }^{8}$ R. W. Patterson and F. H. Abernathy, "Turbulent flow drag reduction and degradation with dilute polymer solutions," J. Fluid Mech. 43, 689 (1970).

${ }^{9}$ D. L. Hunston and J. L. Zakin, "Flow-assisted degradation in dilute polystyrene solutions," Polym. Eng. Sci. 20, 517 (1980).

${ }^{10}$ E. W. Merrill and A. F. Horn, "Scission of macromolecules in dilute solution: Extensional and turbulent flows," Polym. Commun. 25, 144 (1984).

${ }^{11}$ T. Moussa and C. Tiu, "Factors affecting polymer degradation in turbulent pipe flow," Chem. Eng. Sci. 49, 1681 (1994).

${ }^{12}$ H. J. Choi, S. T. Lim, P. Y. Lai, and C. K. Chan, "Turbulent drag reduction and degradation of DNA," Phys. Rev. Lett. 89, 088302 (2002).

${ }^{13}$ V. N. Kalashnikov, "Degradation accompanying turbulent drag reduction by polymer additives," J. Non-Newtonian Fluid Mech. 103, 105 (2002).

${ }^{14}$ C. A. Kim, J. T. Kim, K. Lee, H. J. Choi, and M. S. Jhon, "Mechanical degradation of dilute polymer solutions under turbulent flow," Polymer 41, 7611 (2000).

${ }^{15}$ T. Nakken, M. Tande, and A. Elgsaeter, "Measurements of polymer induced drag reduction and polymer scission in Taylor flow using standard double-gap sample holders with axial symmetry," J. Non-Newtonian Fluid Mech. 97, 1 (2001)

${ }^{16}$ J. H. Sung, S. T. Lim, C. A. Kim, H. J. Chung, and Y. J. Choi, "Mechanical degradation kinetics of polyethylene oxide in turbulent flow," KoreaAust. Rheol. J. 16, 57 (2004).

${ }^{17}$ S. T. Lim, H. J. Choi, S. Y. Lee, J. S. So, and C. K. Chan, " $\lambda$-DNA induced turbulent drag reduction and its characteristics," Macromolecules 36, 5348 (2003)

${ }^{18}$ W. Brostow, H. Ertepinar, and R. P. Singh, "Flow of dilute polymer solutions: Chain conformations and degradation of drag reducers," Macromolecules 23, 5109 (1990).

${ }^{19}$ V. N. Kalashnikov, "Dynamical similarity and dimensionless relations for turbulent drag reduction by polymer additives," J. Non-Newtonian Fluid Mech. 75, 209 (1998).

${ }^{20}$ L. I. Sedov, V. A. Ioselevich, V. N. Pilipenko, and N. G. Vasetskaya, "Turbulent diffusion and degradation of polymer molecules in a pipe and boundary layer," J. Fluid Mech. 94, 561 (1979).

${ }^{21}$ D. T. Walker and W. G. Tiederman, "Turbulent structure in a channel flow with polymer injection at the wall," J. Fluid Mech. 218, 377 (1990).

${ }^{22}$ A. A. Fontaine, H. L. Petrie, and T. A. Brungart, "Velocity profile statistics in a turbulent booundary-layer with slot-injected polymer," J. Fluid Mech. 238, 435 (1992).

${ }^{23}$ T. Moussa, C. Tiu, and T. Sridhar, "Effect of solvent on polymer degradation in turbulent flow," J. Non-Newtonian Fluid Mech. 48, 261 (1993).

${ }^{24}$ A. F. Horn and E. W. Merrill, "Midpoint scission of macromolecules in dilute solution in turbulent flow," Nature (London) 312, 140 (1984).

${ }^{25}$ D. H. Fisher and F. Rodriguez, "Degradation of drag-reducing polymers," J. Appl. Polym. Sci. 15, 2975 (1971).

${ }^{26}$ B. A. Buchholz and A. E. Barron, "The use of light scattering for precise characterization of polymers for DNA sequencing by capillary electrophoresis," Electrophoresis 22, 4118 (2001).

${ }^{27}$ B. A. Buchholz, J. M. Zahn, M. Kenward, G. W. Slater, and A. E. Barron, "Preparing monodisperse high polymers via chain scission in transient extensional flow," Polymer 45, 1223 (2004).

${ }^{28}$ M. T. Islam, S. A. Vanapalli, and M. J. Solomon, "Inertial effects on polymer chain scission in planar elongational cross-slot flow," Macromolecules 37, 1023 (2004).

${ }^{29} \mathrm{P}$. J. Wyatt, "Light-scattering and the absolute characterization of macromolecules," Anal. Chim. Acta 272, 1 (1993).

${ }^{30}$ E. W. Merrill and P. Leopairat, "Scission of non-interpenetrating macromolecules in transient extensional flows," Polym. Eng. Sci. 20, 505 (1980).

${ }^{31}$ T. Q. Nguyen and H. H. Kausch, "Chain extension and degradation in convergent flow," Polymer 33, 2611 (1992).

${ }^{32}$ T. Q. Nguyen and H. H. Kausch, "Influence of nozzle geometry on polystyrene degradation in convergent flow," Colloid Polym. Sci. 269, 1099 (1991).

${ }^{33}$ T. Q. Nguyen and H. H. Kausch, "Chain scission in transient extensional flow kinetics and molecular weight dependence," J. Non-Newtonian Fluid Mech. 30, 125 (1988).

${ }^{34}$ J. A. Odell and A. Keller, "Flow-induced chain fracture of isolated linear macromolecules in solution,” J. Polym. Sci., Part B: Polym. Phys. 24, 1889 (1986).

${ }^{35}$ D. L. Ho, B. Hammouda, and S. R. Kline, "Clustering of polyethylene oxide in water revisited," J. Polym. Sci., Part B: Polym. Phys. 41, 135 (2003).

${ }^{36}$ M. Polverari and T. G. M. van de Ven, "Dilute aqueous polyethylene oxide solutions: Clusters and single molecules in thermodynamic equilibrium," J. Phys. Chem. 100, 13687 (1996).

${ }^{37}$ A. Faraone, S. Magazu, G. Maisano, P. Migliardo, E. Tettamanti, and V. Villari, "The puzzle of polyethylene oxide aggregation in water: Experimental findings," J. Chem. Phys. 110, 1801 (1999).

${ }^{38}$ C. Branca, A. Faraone, S. Magazu, G. Maisano, P. Migliardo, and V. Villari, "Polyethylene oxide: A review of experimental findings by spectroscopic techniques," J. Mol. Liq. 87, 21 (2000).

${ }^{39}$ K. Devanand and J. C. Selser, "Polyethylene oxide does not necessarily aggregate in water," Nature (London) 343, 739 (1990).

${ }^{40} \mathrm{G}$. C. Berry, "Thermodynamic and conformational properties of polystyrene: 1. Light scattering studies on dilute solutions of linear polystyrenes," J. Chem. Phys. 44, 4550 (1966).

${ }^{41}$ P. N. Dunlap and L. G. Leal, "Dilute polystyrene solutions in extensional flows: Birefringence and flow modification," J. Non-Newtonian Fluid Mech. 23, 5 (1987).

${ }^{42}$ J. H. Sung, C. A. Kim, H. J. Choi, B. K. Hur, J. G. Kim, and M. S. Jhon, "Turbulent drag reduction efficiency and mechanical degradation of poly(acrylamide)," J. Macromol. Sci., Phys. B43, 507 (2004).

${ }^{43}$ J. M. J. Den Toonder, A. A. Draad, G. D. C. Kuiken, and F. T. M. Nieuwstadt, "Degradation effects on dilute polymer solutions on turbulent drag reduction in pipe flows," Appl. Sci. Res. 55, 63 (1995).

${ }^{44}$ V. E. Terrapon, Y. Dubief, P. Moin, E. S. G. Shaqfeh, and S. K. Lele, "Simulated polymer stretch in a turbulent flow using Brownian dynamics," J. Fluid Mech. 504, 61 (2004).

${ }^{45}$ C. D. Dimitropoulos, R. Sureshkumar, and A. N. Beris, "Direct numerical simulation of viscoelastic turbulent channel flow exhibiting drag reduction: Effect of the variation of rheological parameters," J. Non-Newtonian Fluid Mech. 79, 433 (1998). 